OPEN ACCESS

Edited by:

Julio Villena,

Centro de Referencia para Lactobacilos (CONICET), Argentina

Reviewed by: María Fernanda Raya Tonetti, Consejo Nacional de Investigaciones Cientificas y Técnicas (CONICET),

Argentina

Md. Aminul Islam,

Tohoku University, Japan

*Correspondence:

Harri Hemilä

harri.hemila@helsinki.fi

tORCID:

Harri Hemilä

orcid.org/0000-0002-4710-307X

Anitra Carr

orcid.org/0000-0002-5890-2977

Elizabeth Chalker

orcid.org/0000-0003-1593-3770

Specialty section:

This article was submitted to Nutritional Immunology,

a section of the journal

Frontiers in Immunology

Received: 01 March 2021 Accepted: 21 April 2021

Published: 10 May 2021

Citation:

Hemilä H, Carr A and Chalker E (2021) Vitamin C May Increase the Recovery Rate of Outpatient

Cases of SARS-CoV-2 Infection by 70\%: Reanalysis of the COVID A to Z Randomized Clinical Trial.

Front. Immunol. 12:674681. doi: 10.3389/fimmu.2021.674681

\section{Vitamin C May Increase the Recovery Rate of Outpatient Cases of SARS-CoV-2 Infection by $70 \%$ : Reanalysis of the COVID A to Z Randomized Clinical Trial}

\author{
Harri Hemilä ${ }^{1 * t}$, Anitra Carr ${ }^{2 \dagger}$ and Elizabeth Chalker ${ }^{3+}$ \\ ${ }^{1}$ Department of Public Health, University of Helsinki, Helsinki, Finland, ${ }^{2}$ Department of Pathology and Biomedical Science, \\ University of Otago, Christchurch, New Zealand, ${ }^{3}$ School of Public Health, University of Sydney, Sydney, NSW, Australia
}

Keywords: ascorbic acid, COVID-19, common cold, quantile treatment effect, randomized controlled trials, SARSCoV-2, statistics, treatment outcome

\section{INTRODUCTION}

Interest in the potential effects of vitamin C for respiratory virus infections traces back to the $1940 \mathrm{~s}$ (1-3). Numerous effects of vitamin $\mathrm{C}$ on the immune system have been demonstrated, which provide the rationale to investigate its effect on infections (4). The most comprehensive summary of the placebo-controlled trials on vitamin $\mathrm{C}$ and respiratory virus infections is the Cochrane review on vitamin $\mathrm{C}$ and the common cold (2).

The Cochrane review identified 31 comparisons that examined the effect of regular vitamin C supplementation of at least $0.2 \mathrm{~g} /$ day on 9745 episodes of respiratory virus infections occurring during the supplementation period. In adults the duration of colds was reduced by $7.7 \%$ (95\% CI $3.7 \%$ to $11.8 \%, \mathrm{P}=0.00018)$ and in children by $14.2 \%(95 \% \mathrm{CI} 7.3 \%$ to $21.1 \%, \mathrm{P}=0.000053)$ [2; Analysis 2.1]. These findings indicate that vitamin $\mathrm{C}$ has physiological effects, though the review did not demonstrate treatment effects on virus infections that had already started (2).

Based on two trials that compared two different vitamin $C$ doses in separate trial arms $(5,6)$, a dose-response relationship for the effect of vitamin $\mathrm{C}$ was indicated, with a prediction that doses of $6-8 \mathrm{~g} /$ day may shorten viral upper respiratory infections by some $20 \%(2,3,7,8)$.

Given the previous research on vitamin C, we read with great interest the report on the COVID A to Z Randomized Clinical Trial (COVID A to Z trial) which investigated the effects of $8 \mathrm{~g} /$ day vitamin $\mathrm{C}$ on the recovery from outpatient SARS-CoV-2 infection (9). However, we unfortunately found several methodological shortcomings in the trial. Here we describe our major concerns and statistical reanalysis of the findings. In addition to the usual care and the vitamin $\mathrm{C}$ arms, the trial included zinc, and zinc with vitamin $\mathrm{C}$ arms. These two arms are not considered in this commentary, however, major problems in the zinc intervention are described in the Supplement. 


\section{METHODOLOGICAL CONCERNS WITH THE COVID A TO Z TRIAL}

The COVID A to Z trial was "stopped early for futility" after recruitment of only 214 patients. The intention had been to recruit 520 patients (9). Stopping early seems unjustified.

The COVID A to Z trial authors described the rationalization for the sample size calculation as follows: "We assumed that the usual care group would achieve a $50 \%$ reduction in symptom severity in a mean (SD) of 6 (3) days and that at least 1 of the other 3 study groups would achieve a 50\% reduction in a mean $(S D)$ of 5 (3) days" (9). This indicates that the authors assumed a 1.0 -day difference in time to a $50 \%$ reduction in symptom severity between the usual care arm and one of the intervention arms. This also indicates that a 1.0-day reduction was considered to be clinically important, otherwise sample size calculations would not have been based on this assumption.

The trial results were reported as follows: "Patients who received usual care without supplementation achieved a 50\% reduction in symptoms in a mean (SD) of 6.7 (4.4) days compared with a mean (SD) of 5.5 (3.7) days for patients receiving ascorbic acid, a mean (SD) of 5.9 (4.9) days for patients receiving zinc gluconate, and a mean (SD) of 5.5 (3.4) days for patients receiving both ascorbic acid and zinc gluconate supplementation" (9).

Thus, time to a $50 \%$ reduction in symptom duration was 1.2 days shorter in the vitamin $\mathrm{C}$ arm than the usual care arm. Given that the observed vitamin C effect was $20 \%$ greater than the expected effect (1.2 vs. 1.0), it is illogical to have stopped the trial early because of "futility" (9). The authors do not explain this paradox: on the one hand, they considered that a 1.0-day reduction in symptom duration is a clinically important difference, yet on the other hand, the observed 1.2-day reduction was futile and justified early termination.

Another substantial concern with the COVID A to Z trial is that it was an "open label trial" (9) without a placebo administered to the control arm. Symptoms of respiratory virus infections are subjective and if there is no blinding there can be systematic bias in either direction between the treatment arms. If a patient believes that vitamin C is effective, he or she might under report symptoms. On the other hand, when patients are given an active intervention, such as vitamin C, they may observe and report symptoms at a lower threshold compared with the usual care arm. Given that the additional cost of a placebo in such a trial is marginal, it is surprising that none was used (9). Furthermore, it is not clear exactly what "open label trial" means and whether the patients were told what they were receiving and how that was communicated. The psychological effects can be quite different depending on how the intervention is described.

Our third concern is about the delay between the onset of symptoms and the initiation of vitamin C. For treating virus diseases, timing to initiation of treatment is important. For example, acyclovir and oseltamivir should be started as soon as possible after the first appearance of symptoms since a delay leads to less efficacy. Previously, long delays were proposed as a potential explanation for some therapeutic trials that did not find vitamin C effective $(2,7)$. In the Methods section, the COVID A to $\mathrm{Z}$ trial authors write: "Patients were randomized ... after a positive diagnosis" (9). However, there is no description of the time between the onset of symptoms and the start of intervention. Given that the analysis was restricted to patients with confirmed COVID-19, it seems probable that the delay between the start of symptoms and the start of treatment was over 24 hours and may have been several days. It is unfortunate that this detail was not described (9).

Our fourth concern is about the distribution of vitamin C dosage: the trial report states " $8000 \mathrm{mg}$ of ascorbic acid [was] (to be divided over 2-3 times per day with meals)" (9). Intestinal absorption of vitamin $\mathrm{C}$ becomes saturated with high doses (10). Because of the decreased absorption, large single doses can cause stomach ailments. Hence, there is a big difference between administering $0.5 \mathrm{~g} /$ hour every hour and administering 4 grams twice a day. The total dose per day is the same, but the adverse effects may be much greater with the latter dosage. For example, in the large trial by Anderson et al. (5), the dosage was "16 tablets (two every hour) on the first day of any illness" (4). Thomas et al. provided no justification for their choice to administer vitamin C 2-3 times per day (9).

Our fifth concern is the lack of commentary around the baseline vitamin C status of the patients. There is great variation in dietary vitamin $\mathrm{C}$ intake and consequently in baseline plasma levels. In this respect vitamin C differs substantially from other drugs, for which the control group uniformly has baseline levels of zero. If baseline levels are high, then supplemental vitamin $\mathrm{C}$ is less likely to have an effect than when baseline levels are low (11).

\section{REANALYSIS OF THE REPORTED FINDINGS}

The difference between the primary outcomes in the vitamin C and usual care patients was not analyzed appropriately for the COVID A to Z trial (9).

The report stated that the duration of symptoms, with the primary endpoint " $50 \%$ reduction in symptoms", was 6.7 days in the usual care arm and 5.5 days in the vitamin C arm (9). The 1.2-day difference was inappropriately ignored on the basis that the associated P-value was not less than 0.05 (12). The focus should be on the estimate and not on the P-value (13).

Moreover, given the great variation in the duration of untreated SARS-CoV-2 infection in the trial (from 2 days to over 3 weeks), the mean difference is a poor measure of the treatment effect. The 1.2-day reduction in symptom duration corresponds to an $18 \%$ reduction on the relative scale which is more informative (14). The $18 \%$ decrease is consistent with the predictions that 6-8 $\mathrm{g} /$ day of vitamin $\mathrm{C}$ will shorten viral respiratory infections by around $20 \%(2,3,7,8)$.

Another approach to analyzing the effect of vitamin $\mathrm{C}$ on the recovery is to use survival analysis. This is not affected by the patients who do not recover during follow-up. The number of patients who recovered with the primary endpoint 
" $50 \%$ reduction in symptoms" on a given day is published in Figure 3 of the report (9). We calculated the rate ratio of recovery between the vitamin $\mathrm{C}$ and usual care arms and found that vitamin $\mathrm{C}$ increased the rate of recovery by $70 \%$ (95\% CI $6.8 \%$ to $170 \%, \mathrm{P}=0.025)$. This direct comparison of vitamin $\mathrm{C}$ and usual care arms was not published by Thomas et al. (9). Had the authors used appropriate statistical methods, they would have found a statistically significant difference. Our calculations and the redrawn survival curve are shown in the Supplement.

We also analyzed the effect of vitamin $\mathrm{C}$ by quantile regression as the quantile treatment effect (QTE) (15-17). In this approach, the distribution of symptom duration in the usual care arm is set on the horizontal axis as percentiles and the difference between the usual care and vitamin C arms is shown as the QTE on the vertical axis (Figure 1). The continuous black line indicates the QTE of vitamin $\mathrm{C}$ administration. For example, at the $60^{\text {th }}$ percentile level, duration was 9 days in the usual care arm, and 6 days in the vitamin $\mathrm{C}$ arm, which corresponds to a QTE of -3 days (95\% CI -4.6 to -3 days; $\mathrm{P}<$ 0.001). The red dotted line indicates the null effect, and the blue dashed line indicates the -1.2 day day mean effect.

The QTE of vitamin C is heterogeneous over the distribution. In the 0 to $29^{\text {th }}$ percentile range, there is no indication of a difference between the arms with a mean duration of 2.9 days in the vitamin $\mathrm{C}$ arm and 2.7 days in the usual care arm. In the $30^{\text {th }}$

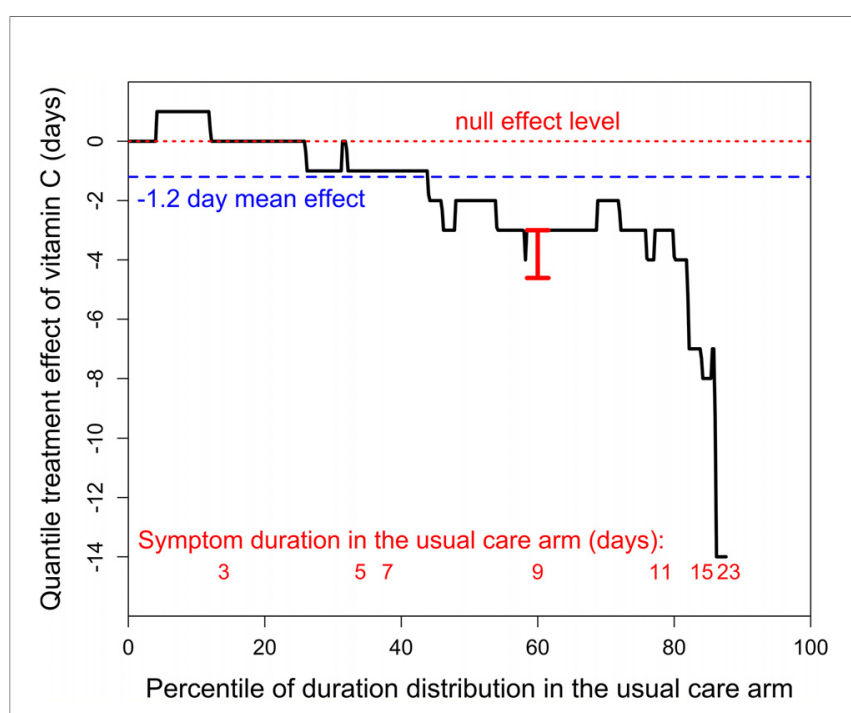

FIGURE 1 | The quantile treatment effect (QTE) of $8 \mathrm{~g}$ /day of vitamin C on the duration of symptoms in outpatient cases of SARS-CoV-2 infection. The horizontal axis shows the distribution of the duration by percentiles. The black stepped line indicates the QTE of vitamin C. The horizontal red dotted line indicates the null effect. The blue dashed line shows the 1.2-day mean effect of vitamin $\mathrm{C}$. The red vertical bar at the $60^{\text {th }}$ percentile indicates the $95 \% \mathrm{Cl}$ (-4.61 to -3 days; $\left.t=7.3 \mathrm{P}=10^{-10}\right)$, calculated with the fit.crq procedure of the R-statistical software (15-17). The red numbers at the bottom indicate the lowest percentile level for the odd number of days of duration of SARS-CoV-2 infection in the usual care arm. For example, 9-10 day infections cover the percentile range from $58^{\text {th }}$ to $76^{\text {th }}$, which corresponds to 9 patients, as the total number of usual care patients was 50 . The QTE curve is stopped at the $88^{\text {th }}$ percentile since 6 patients in the usual care group were censored, i.e., did not recover by the end of the follow-up. See extracted data in Table S1, and our calculations and the redrawn recovery curve in the Supplementary File. to $59^{\text {th }}$ percentile range, mean duration in the vitamin $\mathrm{C}$ arm was 1.6 days (26\%) shorter (4.6 vs 6.2 days), and in the $60^{\text {th }}$ to $88^{\text {th }}$ percentile range, mean duration in the vitamin $\mathrm{C}$ arm was 4.0 days (34\%) shorter (7.6 vs 11.6 days), see Table S2 in the Supplement. Thus, the 1.2 day (18\%) overall mean effect may be a substantial underestimate of the potential effect of vitamin C on longer cases of SARS-CoV-2 infection, which are of particular concern $(18,19)$. Vitamin $\mathrm{C}$ has also been shown to have a greater effect on more severely ill patients in some previous studies (20-23).

\section{DISCUSSION}

There is strong evidence that vitamin $\mathrm{C}$ can shorten the duration of respiratory virus infections $(2,3,5-8)$. There are many different respiratory viruses, and their distribution varies over time and place. The respiratory viruses covered in many trials of vitamin $\mathrm{C}$ are non-specific and so it is unlikely that the benefits of vitamin $\mathrm{C}$ are constrained to a particular respiratory virus or virus group. Thus, it seems likely that vitamin $\mathrm{C}$ may also have an effect on the SARS-CoV-2 coronavirus $(11,24)$.

The COVID A to $\mathrm{Z}$ trial is important as it focused specifically on SARS-CoV-2 coronavirus patients and examined a high dose of vitamin $\mathrm{C}$ which was previously predicted to reduce the duration of respiratory virus infections by about $20 \%(2,3,7,8)$. Unfortunately, there are several limitations in the trial methods and the trial was terminated early, even though the observed benefit from vitamin C was greater than assumed in the sample size calculations.

Regrettably, another recent trial on vitamin $\mathrm{C}$ published in JAMA also contained analysis errors (25), and another two recent reviews on vitamin $\mathrm{C}$ and respiratory infections were misleading $(26,27)$.

Our reanalysis of the COVID A to Z Trial results indicates that there is a statistically significant difference in the recovery rate between the vitamin $\mathrm{C}$ and usual care arms $(\mathrm{P}=0.025)$, and the analysis of the quantile treatment effect indicates that there may be around $30 \%$ reduction in symptom duration in patients with the longest symptoms. These findings indicate a need for methodologically sound trials with larger numbers of patients to investigate the treatment effects of vitamin C against SARS-CoV-2.

\section{AUTHOR CONTRIBUTIONS}

$\mathrm{HH}$ originated the study, carried out the statistical analyses, and wrote the draft. AC and EC participated in the revision of the manuscript. All authors contributed to the article and approved the submitted version.

\section{SUPPLEMENTARY MATERIAL}

The Supplementary Material for this article can be found online at: https://www.frontiersin.org/articles/10.3389/fimmu.2021. 674681/full\#supplementary-material 


\section{REFERENCES}

1. Markwell NW. Vitamin C in the Prevention of Colds. Med J Aust (1947) 2:777-8. doi: 10.5694/j.1326-5377.1947.tb74386.x

2. Hemilä H, Chalker E. Vitamin C for Preventing and Treating the Common Cold. Cochrane Database Syst Rev (2013) 31:CD000980. doi: 10.1002/ 14651858.CD000980.pub4

3. Hemilä H. Vitamin C and Infections. Nutrients (2017) 9:339. doi: 10.3390/ nu9040339

4. Carr AC, Maggini S. Vitamin C and Immune Function. Nutrients (2017) 9:1211. doi: 10.3390/nu9111211

5. Anderson TW, Suranyi G, Beaton GH. The Effect on Winter Illness of Large Doses of Vitamin C. Can Med Assoc J (1974) 111:31-6.

6. Karlowski TR, Chalmers TC, Frenkel LD, Kapikian AZ, Lewis TL, Lynch JM. Ascorbic Acid for the Common Cold: A Prophylactic and Therapeutic Trial. JAMA (1975) 231:1038-42. doi: 10.1001/jama.1975.03240220018013

7. Hemilä H. Vitamin C Supplementation and Common Cold Symptoms: Factors Affecting the Magnitude of the Benefit. Med Hypotheses (1999) 52:171-8. doi: 10.1054/mehy.1997.0639

8. Hemilä H, Vitamin C. The Placebo Effect, and the Common Cold: A Case Study of How Preconceptions Influence the Analysis of Results [Comments in (1996) 49:1085-87]. J Clin Epidemiol (1996) 49:1079-84. doi: 10.1016/08954356(96)00189-8

9. Thomas S, Patel D, Bittel B, Wolski K, Wang Q, Kumar A, et al. Effect of High-Dose Zinc and Ascorbic Acid Supplementation vs Usual Care on Symptom Length and Reduction Among Ambulatory Patients With SARSCoV-2 Infection: The COVID A to Z Randomized Clinical Trial. JAMA Netw Open (2021) 4:e210369. doi: 10.1001/jamanetworkopen.2021.0369

10. Padayatty SJ, Sun H, Wang Y, Riordan HD, Hewitt SM, Katz A, et al. Vitamin C Pharmacokinetics: Implications for Oral and Intravenous Use. Ann Intern Med (2004) 140:533-7. doi: 10.7326/0003-4819-140-7-200404060-00010

11. Carr AC, Rowe S. The Emerging Role of Vitamin C in the Prevention and Treatment of COVID-19. Nutrients (2020) 12:3286. doi: 10.3390/ nu12113286

12. Amrhein V, Greenland S, McShane B. Scientists Rise Up Against Statistical Significance. Nature (2019) 567:305-7. doi: 10.1038/d41586-019-00857-9

13. Schmidt M, Rothman KJ. Mistaken Inference Caused by Reliance on and Misinterpretation of a Significance Test. Int J Cardiol (2014) 177:1089-90. doi: 10.1016/j.ijcard.2014.09.205

14. Hemilä H. Duration of the Common Cold and Similar Continuous Outcomes Should be Analyzed on the Relative Scale: A Case Study of Two Zinc Lozenge Trials. BMC Med Res Methodol (2017) 17:82. doi: 10.1186/s12874-017-0356-y

15. Koenker R. Quantile Treatment Effects. In: Quantile Regression. Cambridge: Cambridge University Press (2005). p. 26-32.
16. Koenker R. Quantreg: Quantile Regression. R Package Version 5.67 (2020). Available at: https://CRAN.R-project.org/package=quantreg.

17. Hong HG, Christiani DC, Li Y. Quantile Regression for Survival Data in Modern Cancer Research: Expanding Statistical Tools for Precision Medicine. Precis Clin Med (2019) 2:90-9. doi: 10.1093/pcmedi/pbz007

18. Carfi A, Bernabei R, Landi F. Gemelli Against COVID-19 Post-Acute Care Study Group. Persistent Symptoms in Patients After Acute COVID-19. JAMA (2020) 324:603-5. doi: 10.1001/jama.2020.12603

19. Davis HE, Assaf S, Lisa McCorkell L, Wei H, Low RJ, Re'em Y, et al. Characterizing Long COVID in an International Cohort: 7 Months of Symptoms and Their Impact. MedRxiv (2020).

20. Hemilä H. Vitamin C may Alleviate Exercise-Induced Bronchoconstriction: A Meta-Analysis. BMJ Open (2013) 3:e002416. doi: 10.1136/bmjopen-2012-002416

21. Hemilä H, Chalker E. Vitamin C May Reduce the Duration of Mechanical Ventilation in Critically Ill Patients: A Meta-Regression Analysis. J Intensive Care (2020) 8:15. doi: 10.1186/s40560-020-0432-y

22. Hemilä H, Chalker E. Vitamin C Can Shorten the Length of Stay in the ICU: A Meta-Qnalysis. Nutrients (2019) 11:708. doi: 10.3390/nu11040708

23. Zhang J, Rao X, Li Y, Zhu Y, Liu F, Guo G, et al. Pilot Trial of High-Dose Vitamin C in Critically Ill COVID-19 Patients. Ann Intensive Care (2021) 11:5. doi: 10.1186/s13613-020-00792-3

24. Hemilä H, de Man AME. Vitamin C and COVID-19. Front Med (2021) 7:559811. doi: 10.3389/fmed.2020.559811

25. Hemilä H, Chalker E. Reanalysis of the Effect of Vitamin C on Mortality in the CITRIS-ALI Trial: Important Findings Dismissed in the Trial Report. Front Med (Lausanne) (2020) 7:590853. doi: 10.3389/fmed.2020.590853

26. Hemilä H, Chalker E. Commentary: Vitamin C Supplementation for Prevention and Treatment of Pneumonia. Front Med (Lausanne) (2021) 7:595988. doi: 10.3389/fmed.2020.595988

27. Hemilä H and Chalker E. Commentary: The Long History of Vitamin C: From Prevention of the Common Cold to Potential Aid in the Treatment of COVID-19. Front Immunol (2021) 12:659001. doi: 10.3389/fimmu.2021.659001

Conflict of Interest: The authors declare that the research was conducted in the absence of any commercial or financial relationships that could be construed as a potential conflict of interest.

Copyright (c) 2021 Hemilä, Carr and Chalker. This is an open-access article distributed under the terms of the Creative Commons Attribution License (CC BY). The use, distribution or reproduction in other forums is permitted, provided the original author(s) and the copyright owner(s) are credited and that the original publication in this journal is cited, in accordance with accepted academic practice. No use, distribution or reproduction is permitted which does not comply with these terms. 